\title{
Characterization of retinal regeneration in adult zebrafish following multiple rounds of phototoxic lesion
}

\author{
Alexandra H Ranski ${ }^{1}$, Ashley C Kramer ${ }^{1}$ ， Gregory W Morgan ${ }^{1}$ ， Jennifer L Perez ${ }^{1}$, Ryan Thummel $^{\text {Corresp. }}{ }^{1}$ \\ ${ }^{1}$ Department of Ophthalmology, Visual, and Anatomical Sciences, Wayne State University School of Medicine, Detroit, MI \\ Corresponding Author: Ryan Thummel \\ Email address: rthummel@med.wayne.edu
}

Müller glia in the zebrafish retina respond to retinal damage by re-entering the cell cycle, which generates large numbers of retinal progenitors that ultimately replace the lost neurons. In this study we compared the regenerative outcomes of adult zebrafish exposed to one round of phototoxic treatment with adult zebrafish exposed to six consecutive rounds of phototoxic treatment. We observed that Müller glia continued to re-enter the cell cycle to produce clusters of retinal progenitors in zebrafish exposed to multiple rounds of phototoxic light. Some abnormalities were noted, however. First, we found that retinas exposed to multiple rounds of damage exhibited a greater loss of photoreceptors at 36 hours of light damage than retinas that were exposed to their first round of light damage. In addition, we found that Müller glia appeared to have an increase in the acute gliotic response in retinas exposed to multiple rounds of light treatment. This was evidenced by cellular hypertrophy, changes in GFAP cellular localization, and transient increases in stat3 and gfap expression. Finally, following the sixth round of phototoxic lesion, we observed a significant increase in mis-localized HuC/D-positive amacrine and ganglion cells in the inner plexiform layer and outer retina, and a decreased number of regenerated blue cone photoreceptors. These data add to recent findings that retinal regeneration in adult zebrafish occurs concomitant with Müller glia reactivity and can result in the generation of aberrant neurons. These data are also the first to demonstrate that Müller glia appear to modify their phenotype in response to multiple rounds of phototoxic lesion, exhibiting an increase in acute gliosis while maintaining a remarkable capacity for long-term regeneration of photoreceptors. 


\section{Characterization of retinal regeneration in adult zebrafish following multiple}

3 Alexandra H. Ranski ${ }^{1}$, Ashley C. Kramer ${ }^{1}$, Gregory W. Morgan ${ }^{1}$, Jennifer L. Perez ${ }^{1}$, Ryan

4 Thummel ${ }^{1,2 *}$

$5{ }^{1}$ Wayne State University School of Medicine, Department of Ophthalmology, Visual, and

6 Anatomical Sciences, Detroit, MI

7

8

\section{Email Addresses:}

Alexandra H. Ranski aranski@oakland.edu

Ashley C. Kramer

ashley.kramer3@med.wayne.edu

Gregory W. Morgan

gwmorgan@uw.edu

Jennifer L. Perez

jlp232@pitt.edu

Ryan Thummel

rthummel@med.wayne.edu

\section{*Corresponding Author}

Ryan Thummel, Ph.D.

Department of Ophthalmology, Visual, and Anatomical Sciences

Wayne State University School of Medicine

Detroit, MI

rthummel@med.wayne.edu

(313) $577-7762$

Running title: Multiple rounds of phototoxic lesion 
25

26

27

28

29

30

31

\section{ABSTRACT}

Müller glia in the zebrafish retina respond to retinal damage by re-entering the cell cycle, which generates large numbers of retinal progenitors that ultimately replace the lost neurons. In this study we compared the regenerative outcomes of adult zebrafish exposed to one round of phototoxic treatment with adult zebrafish exposed to six consecutive rounds of phototoxic treatment. We observed that Müller glia continued to re-enter the cell cycle to produce clusters of retinal progenitors in zebrafish exposed to multiple rounds of phototoxic light. Some abnormalities were noted, however. First, we found that retinas exposed to multiple rounds of damage exhibited a greater loss of photoreceptors at 36 hours of light damage than retinas that were exposed to their first round of light damage. In addition, we found that Müller glia appeared to have an increase in the acute gliotic response in retinas exposed to multiple rounds of light treatment. This was evidenced by cellular hypertrophy, changes in GFAP cellular localization, and transient increases in stat 3 and gfap expression. Finally, following the sixth round of phototoxic lesion, we observed a significant increase in mis-localized HuC/D-positive amacrine and ganglion cells in the inner plexiform layer and outer retina, and a decreased number of regenerated blue cone photoreceptors. These data add to recent findings that retinal regeneration in adult zebrafish occurs concomitant with Müller glia reactivity and can result in the generation of aberrant neurons. These data are also the first to demonstrate that Müller glia appear to modify their phenotype in response to multiple rounds of phototoxic lesion, exhibiting an increase in acute gliosis while maintaining a remarkable capacity for long-term regeneration of photoreceptors. 


\section{INTRODUCTION}

Adult zebrafish (Danio rerio) have the ability to regenerate numerous different tissues

including cardiac tissue, retinal tissue, and large portions of their appendages (i.e. fins) (Chablais et al. 2011; Gonzalez-Rosa et al. 2011; Poss et al. 2002; Schnabel et al. 2011). These capabilities are contrasted in mammalian species by the formation of scars in these tissues rather than complete regeneration. Though the aforementioned tissues in the zebrafish appear to harbor the same populations of resident cell types as their mammalian counterparts, i.e. Müller glia in the retina and fibroblasts in the skin, their response to damaging stimuli is quite different. This difference in response to damage results in regeneration in one species but gliotic/fibrotic scar formation in the other. In addition, there is evidence that multiple tissues in the zebrafish rely on shared pathways for these regenerative capabilities. For example, the genes mps 1 and $h s p d l$, which are necessary for proper regeneration in the heart and fin, are also upregulated in the regenerating zebrafish retina, indicating that although the details of regenerative mechanisms in each tissue might be different, there may be overlapping genetic networks at play in zebrafish tissues that allow them this unique regenerative quality (Qin et al. 2009).

Müller glial cells are the resident cell type in the vertebrate retina whose homeostatic function is neuronal support, including neurotransmitter recycling and production of glutamine synthetase (Bringmann et al. 2006) In both the zebrafish and the mammalian retina, photoreceptor ablation by intense light exposure initially results in Müller cell gliosis, including hypertrophy and upregulation of gfap (Thomas 2015). However, in the zebrafish, this response is then down-regulated as Müller glia generate large numbers of retinal progenitors (Thummel et al. 2008a). A similar phenomenon also occurs in the zebrafish skin, as a laser-induced full-thickness wound results in initial scar formation that regresses as the epidermis is restored (Richardson et 
71 al. 2013). These data suggest that permissive species, such as zebrafish, use the complementary

72 roles of a reactive state, as seen in retinal gliosis and skin fibrosis, with a subsequent progenitor

73 proliferation to successfully restore damaged tissue.

74 Multiple studies have indicated that by inhibiting some of the pathways that zebrafish use

75 to regenerate their tissues, the scar formation seen in mammals can be mimicked. For example,

76 inhibition of cardiomyocyte proliferation by mps 1 inhibition resulted in persistent scar formation

77 in the heart (Poss et al. 2002). Similarly, inhibition of Fgf signaling during full-thickness wound

78 healing also resulted in persistent scar formation in the epidermis (Richardson et al. 2013).

79 Lastly, multiple rounds of amputation/regeneration of fin tissue resulted in normal regeneration

80 of new tissue, but an increased deposition of collagen in the underlying and existing tissue

81 (Azevedo et al. 2011). These studies support growing evidence that damaged tissue initially

82 results in a gliotic/fibrotic response, which is then followed by a regenerative response.

83 Furthermore, while many genes that control the proliferation response in these tissues are known,

84 signals that affect gliosis/fibrosis versus stemness in permissive species like zebrafish are largely

85 unknown. Additionally, the response of Müller glia to more than one round of phototoxic

86 damage has not yet been described in the literature to the best of our knowledge. Therefore, the

87 purpose of this study was to determine whether multiple rounds of light exposure would lead to a 88 gliotic response and/or failure to regenerate.

89 Here we characterize, for the first time, the response of the adult zebrafish retina to

90 multiple rounds of damaging stimuli, with a special interest in both the gliotic and stem cell

91 responses of the resident Müller glia. We first hypothesized that upon consecutive damage to the

92 zebrafish retina, that the Müller glia would respond randomly to the damage event, and not

93 recruit the exact same population of Müller glia for both the first and the second light exposures. 
94 We exposed dark-adapted adult albino zebrafish to four days of intense light to destroy the rod 95 and cone photoreceptors and then observed a full regeneration of the retinal tissue by 28 days 96 after light onset. Next, we exposed the same fish to a second round of light damage. Consistent 97 with our hypothesis, we found that not all of Müller glia that re-entered the cell cycle during the 98 first round of regeneration responded again during the second round of regeneration.

99 Secondly, we hypothesized that treating the retina with intense light multiple times would 100 result in Müller glia with a more robust gliotic phenotype. When we analyzed the regenerative 101 potential of the retina following 6 rounds of phototoxic treatment, we found that Müller glia still 102 re-entered the cell cycle to produce retinal progenitors. However, we observed signs of increased 103 gliosis among the Müller glia as these events occurred, including an upregulation of gfap and a 104 redistribution of GFAP protein, cellular hypertrophy, and changes in gene expression associated 105 with persistent gliosis. In addition, we observed significantly increased aberrant localization of 106 inner retinal neurons following six rounds of damage and a decreased number of blue cones.

107 Therefore, these studies illustrate that subsets of Müller glia are capable of response to multiple 108 rounds of photolytic damage and regenerate photoreceptors. However, increasing the number of 109 damage and response events required of this Müller glia stem cell population results in an 110 increase in the acute gliotic response. 


\section{MATERIALS AND METHODS}

\section{Fish Maintenance}

114

115

Two fish lines were used for these studies: adult (9-18 months) albino (alb) and adult (9-

11618 months) $\operatorname{Tg}(g f a p: e g f p) / a l b$ zebrafish. Unless otherwise noted, fish were maintained under a

117 daily light cycle of 14 hours light (250 lux):10 hours dark at $28.5^{\circ} \mathrm{C}$ (Westerfield 1995) and fed a 118 combination of flake food and brine shrimp. The Institutional Animal Care and Use Committee 119 at Wayne State University approved the phototoxic lesion protocol used in this study (Protocol \# 120 16-01-037).

121

\section{Intense Light Exposure}

A photolytic damage model was used to destroy rod and cone photoreceptors (Thomas et

124 al. 2012). First, 5 adult albino fish per treatment group were dark-adapted for 10 days. For the

125 untreated fish ( $0 \mathrm{~h}$ control group), eye tissue was harvested after the 10 day dark-adaptation. For

126 fish that were exposed to photolytic damage to photoreceptors, following the 10 day dark-

127 adaption, animals were first exposed to approximately 100,000 lux for 30 minutes using a

128 broadband light source (Thomas et al. 2012). Next, fish were transferred to 1.8-liter tanks and 129 exposed to approximately 10,000 lux of light from four, 250 Watt halogen lamps (Thomas et al.

130 2012). Fish were subjected to constant light exposure for up to four days, and then transferred

131 back to standard light:dark conditions. For fish that underwent 1 round of light treatment ( $1 \mathrm{x}$

132 treatment group), eye tissue was collected at the time points indicated. These animals were 9-10

133 months of age. For fish that underwent multiple rounds of light treatment (6x treatment group),

134 following the first light treatment, fish were allowed to recover for 28 days, dark-adapted for 10

135 days, and then exposed to another round of light lesion as described above. This was repeated for 
136 a total of 6 rounds of light treatment and 28 day recovery period. As these fish were now 18

137 months of age, the experiment was terminated after 6 rounds in order to not exceed our normal

138 window of 9-18 months of age for light treatments.

139

140

BrdU Incorporation

141

For the BrdU study, adult (9-18 months) albino fish were light treated for 24 hours. Next,

142 fish were transferred to a $1 \mathrm{~L}$ solution containing $0.66 \mathrm{~g}$ of $\mathrm{NaCl}, 0.1 \mathrm{~g}$ Neutral Regulator, and $1.5 \mathrm{~g}$

143 BrdU (SeaChem Laboratories, Inc. Stone Mountain, GA; Sigma). After 24 hours, these fish were

144 transferred to normal light:dark conditions for 28 days, dark-adapted for 10 days, and then

145 exposed to another round of light lesion as described above. The timeframe for BrdU incubation

146 was based on previous studies which indicated that all participating Müller glia re-enter the cell

147 cycle within this window, starting at $\sim 31 \mathrm{hpL}$, and that the first daughter cell is visible at $48 \mathrm{hpL}$

148 (Kassen et al. 2008; Thummel et al. 2008a; Vihtelic \& Hyde 2000). Thirty-six hours after

149 starting the second treatment, fish were euthanized and whole eyes were enucleated and

150 processed for immunohistochemistry. The percentage of Müller glia in each group (BrdU+,

151 PCNA+, double-positive) were calculated within a linear distance of 300 microns. The Kruskal-

152 Wallis nonparametric $\mathrm{H}$ test was used to assess whether there were significant differences

153 between the groups at the 0.05 significance level.

154

155 Immunohistochemistry and Confocal Microscopy

156 Immunohistochemistry was performed as previously described (Thummel et al. 2008a).

157 Briefly, 5 whole eyes from each experimental group were harvested from sacrificed adult (9-18

158 months) animals and were fixed in 9:1 ethanolic formaldehyde (100\% ethanol: 37\% 
159 formaldehyde) overnight at $4{ }^{\circ} \mathrm{C}$. Eyes were cryopreserved, embedded in freezing medium, and

160 retinal sections $(16 \mu \mathrm{M})$ containing or immediately surrounding the optic nerve were collected

161 on Superfrost Plus glass slides (Fisher Scientific, Pittsburgh, PA). Two different

162 immunohistochemistry protocols were used for the primary antibody incubation. Standard

163 immunohistochemistry was performed as previously described (Thummel et al. 2008a) using the

164 following primary antibodies: mouse Zpr-1 antibody (Zebrafish International Resource Center,

165 Eugene, OR; 1:200), rabbit anti-Rhodopsin antisera (gift from David Hyde; 1:5,000), rabbit anti-

166 GFP (Abcam; 1:1,500), mouse anti-PCNA (Sigma; 1:1,000), rabbit anti-Blue opsin (gift from

167 David Hyde; 1:500), rabbit anti-UV opsin (gift from David Hyde; 1:1000), mouse anti-

168 Glutamine Synthetase (Chemicon; 1:500), and mouse 4C4 (gift from Peter Hitchcock; 1:250).

169 For antibodies requiring antigen retrieval, the following procedure was used. Slides were

170 preheated to $55^{\circ} \mathrm{C}$ on a slide warmer while a glass coplin jar was preheated in an Oster 6-quart

171 Digital Food Steamer for 30 minutes. Sodium Citrate Buffer (10 mM Sodium

172 Citrate/1XPBS/0.05\% Tween-20, $\mathrm{pH}$ 6.0), heated in a microwave until boiling, was poured into

173 the coplin jar and the slides were immediately placed in the buffer solution. After 30 minutes, the

174 coplin jar was removed from the steamer and allowed to cool at room temperature for 30

175 minutes. The slides were removed and the tissue was outlined with a Pap Pen (Daido Sangyo,

176 Tokyo, Japan). Slides were rinsed three times for 5 minutes with 1XPBS/.5\% Triton X-100.

177 Retinal sections were covered with a blocking solution of 1 XPBS/.5\% Triton X-100/20\% sheep

178 serum for 1 hour. Sections were incubated overnight at $4{ }^{\circ} \mathrm{C}$ in primary antibody diluted into

179 1XPBS/.5\% Triton X-100/2\% sheep serum. The primary antibodies and antisera requiring use of

180 this protocol were: mouse anti-HuC/D (Invitrogen; 1:50) and rabbit anti-GFAP

181 (DakoCytomation; 1:500). 
183 times for 10 minutes with PBS/.5\% Triton X-100 at room temperature. Sections were then

184 incubated in the secondary antibody solution diluted in $1 \mathrm{XPBS} / .5 \%$ Triton $\mathrm{X}-100 / 2 \%$ sheep

185 serum for 1 hour. After secondary antibody incubation, sections were rinsed in 1XPBS/.5\%

186 Triton X-100 3 times for 10 minutes. Slides were covered with a coverslip using ProLong Gold

187 (Molecular Probes, Eugene, OR). Secondary antibodies included AlexaFluor-conjugated 488,

188594 and 647 goat anti-primary (1:500, Life Technologies, Grand Island, NY). Nuclei were

189 stained using TO-PRO-3 (TP3; 1:750; Life Technologies, Grand Island, NY) or DAPI.

190 Confocal microscopy was performed with a Leica TCS SP8 confocal microscope. Z-

191 stacked images were taken within a $4 \mu \mathrm{M}$ thickness with $0.5 \mu \mathrm{M}$ between slices. Quantification

192 of retinal cell number was performed by manual count of a 300 micron linear distance on the

193 central dorsal retina using 5 captured images in each group of biological replicates. The

194 differences between groups were analyzed by a one-way ANOVA if three groups were compared

195 as seen in all photoreceptor quantifications and the quantification of mis-localized HuC/D+

196 neurons. A Student's T-test was performed using $\mathrm{p}<0.05$ as a statistical cut-off if only two

197 groups were compared as in the comparison of PCNA+ Müller glia at 36 hours post light (hpL)

198 and qRT-PCR results.

199

200 Quantitative Real-Time PCR

201 Retinal tissue was isolated from control and experimental groups at $36 \mathrm{hpL}, 72 \mathrm{hpL}$, and

20228 days post light (dpL). Pools of 5 retinas were collected in biological triplicate. Tissue was

203 collected into $1 \mathrm{~mL}$ of Trizol, manually homogenized, and frozen in Trizol at -80 . RNA was

204 isolated using the Trizol Reagent, per manufacturer's instructions (Invitrogen). DNase treatment 
205 was not performed. RNA was quantified and assessed for purity using a nanodrop. 1 $\mu$ g RNA was

206 used as the input for cDNA synthesis, which was performed using the manufacturer's protocol

207 with random oligos and Superscript II polymerase (Invitrogen, 18064). cDNA was diluted 1:25

208 in nuclease free $\mathrm{H}_{2} \mathrm{O}$ and $2 \mu \mathrm{L}$ was used in the following reaction. Quantitative Real-Time PCR

209 (qPCR) was carried out in technical and biological triplicate in a $10 \mu \mathrm{L}$ reaction using the SYBR

210 green reagent (Applied Biosystems, \#4309155) on a CFX Connect Real-Time System (Bio-Rad).

211 Cycling conditions were as follows: step $1.95^{\circ} \mathrm{C}$ hold for $10 \mathrm{~min}$, step $2.95^{\circ} \mathrm{C}$ denature for $15 \mathrm{sec}$,

$21260-63^{\circ} \mathrm{C}$ anneal/extend for $1 \mathrm{~min}$ (cycle step 2. 39 times), step 3.65-95 $\mathrm{C}$ melt-curve increasing by

$2130.5^{\circ} \mathrm{C}$ every $5 \mathrm{sec}$. Primers were designed to span introns with the exception of the gpia primer

214 pair (BIO-RAD Assay ID:qDreCED0007433) which was used as the endogenous reference gene.

215 Primer sequences are displayed in Table 1. PCR products were not sequenced to confirm

216 identity; however, the melt curve was analyzed to determine single peaks for each primer set.

217 Quantification of fold-change in expression was performed between control (1x) and

218 experimental (6x) groups using the Livak $2^{-\Delta \Delta C(t)}$ method with a $\mathrm{p}<0.05$ for statistical cut-off.

219 (Pfaffl 2001). Briefly, the mean CT for each gene was normalized against the gpia mean CT

220 endogenous reference to obtain a $\Delta \mathrm{CT}$ value. The $\Delta \mathrm{CT}$ value of the $6 \mathrm{x}$ group was averaged

221 across the biological triplicate and then normalized against the $1 \mathrm{X}$ group, giving the $\Delta \Delta \mathrm{CT}$ value.

222 Statistical differences between groups were determined using a student's T-test from the

223 normalized $\Delta \Delta \mathrm{CT}$ values. Finally, $\Delta \Delta \mathrm{CT}$ values were converted to log 2 -fold changes in gene

224 expression and graphed.

225 


\section{RESULTS}

\section{Evidence that the exact same population of Müller glia do not re-enter the cell cycle}

\section{8 following each light damage event.}

The timeline of light-induced damage and regeneration of the zebrafish retina has

230 previously been described (Kassen et al. 2007; Lenkowski \& Raymond 2014; Thomas et al.

231 2012; Thummel et al. 2008a; Vihtelic \& Hyde 2000). Briefly, photoreceptor apoptosis peaks at

$23224 \mathrm{hpL}$, followed by Müller glia cell cycle re-entry at 31-36 hpL. The resultant progenitors

233 continue to proliferate over the next 3 days as they migrate from the inner nuclear layer (INL) to

234 the outer nuclear layer (ONL), where they begin to differentiate into new photoreceptors during

235 the following week. Previous reports showed that $\sim 50 \%$ of the total population of Müller glia re-

236 enter the cell cycle during the photolesion paradigm (Thummel et al., 2008b). Given that this

237 study used multiple rounds of phototoxic treatment, we were first interested in testing whether

238 the exact same subset of Müller glia that acted as stem cells following one round of damage

239 would respond in the same way a second time. To examine this, adult albino zebrafish were

240 subjected to two rounds of light damage. During the first round, Müller glia that re-entered the

241 cell cycle were permanently marked with BrdU incorporation during the S phase of the cell

242 cycle. During the second round of Müller glia cell re-entry at $36 \mathrm{hpL}$, retinal sections were triple

243 immunolabeled with antibodies against BrdU (S phase during the first round), PCNA (G1 $\rightarrow \mathrm{S}$

244 phase during the second round), and GFAP (all Müller glia). We observed that 24\% of GFAP-

245 positive Müller glia were only immunolabeled with anti-BrdU (Fig. 1, arrowheads), marking

246 cells that re-entered the cell cycle following the first round of damage, but not the second.

247 Conversely, 40\% of GFAP-positive Müller glia were only immunolabeled with anti-PCNA (Fig.

248 1, arrows), indicating cells that initiated cell cycle re-entry only during the second round of light 
249 damage, but not the first. Finally, 35\% of GFAP-positive Müller glia were co-immunolabeled

250 with anti-BrdU, and anti-PCNA (Fig.1, double arrowheads), marking cells that re-entered the cell

251 cycle during both rounds of regeneration. A Kruskal-Wallis nonparametric H-test determined

252 that there were not significant differences between these groups $(p=0.101)$. These data suggest

253 that the exact same population of Müller glia do not re-enter the cell cycle following each

254 individual light damage event.

255

256

257

Multiple rounds of light damage leads to a greater loss of photoreceptors and increased Müller cell gliosis, but a normal Müller glial and progenitor cell proliferation

Next, we compared photoreceptor numbers and proliferation in untreated control animals

259

with animals exposed to 1 round $(1 \mathrm{x})$ and 6 rounds $(6 \mathrm{x})$ of light treatment at two intermediate time points, 36 and $72 \mathrm{hpL}$ (Thummel et al. 2008a). At $36 \mathrm{hpL}$, a one-way ANOVA analysis was

performed among all photoreceptors quantified and revealed that there were statistically significant differences among the groups $(\mathrm{p}<0.0001)$. Notably, a post-hoc test also revealed statistical differences between the $1 \mathrm{x}$ and $6 \mathrm{x}$ groups. Specifically, 6x light treated retinas exhibited decreased Rhodopsin immunolocalization (Fig. 2B-D), rod photoreceptor nuclei in the outer nuclear layer (Fig. 2Q; $<<0.05$ ) and significantly fewer numbers of long-single (blue) cones (Fig. 2H-J, S; p<0.02) and short-single (UV) cones (Fig. 2K-M, T; p<0.006) when compared with the $1 \mathrm{x}$ treated retinas. Zpr-1 positive double cones were nearly completely destroyed in both groups (Fig. 2E-G, R; p>0.05). Additionally, 6x retinas showed a significantly higher number of 4C4-positive microglia/macrophages compared with 1x retinas (Fig. 2O-P; Average number of 13.8 vs. 46.2 per $300 \mu \mathrm{m}$, respectively; $\mathrm{p}<0.01$ ), which is consistent with the visually more

271 pronounced cellular debris observed in the 6x retinas (Fig. 2G, J, M; arrows). These data suggest 
272 that retinas that have undergone $6 x$ rounds of damage and regeneration exhibit increased

273 photoreceptor loss to a subsequent light insult and exhibited an increased inflammatory response.

274 Increased photoreceptor loss would predict a higher percentage of Müller glia to re-enter

275 the cell cycle at $36 \mathrm{hpL}$ (Thomas et al. 2012). Contrary to this prediction, we observed that the

276 percentage of Müller cells that re-entered the cell cycle was not significantly different between

277 the $1 \mathrm{x}$ and $6 \mathrm{x}$ groups (Fig. 3B-C, J). In addition, at $72 \mathrm{hpL}$, both $1 \mathrm{x}$ and $6 \mathrm{x}$ retinas show Müller

278 glia surrounded by clusters of PCNA-positive progenitors migrating to the outer nuclear layer

279 (ONL; Fig. 4E-F), suggesting that progenitor amplification and migration was not severely

280 altered in 6x retinas. However, retinas from the 6x groups did show signs of increased Müller

281 cell gliosis, including unorganized, hypertrophied Müller glia with a significant upregulation of

282 glial fibrillary acidic protein (gfap) mRNA expression (Fig. 3K), and thickened GFAP-positive

283 appendages in the photoreceptor layer at $36 \mathrm{hpL}$ (Fig. 3E-F, H-I). Expression of signal

284 transducer and activator of transcription 3 (stat3), one of the earliest molecular markers of

285 retinal stress, was also significantly upregulated (Fig. 3K) (Gorsuch \& Hyde 2014; Nicolas et al.

286 2013). At $72 \mathrm{hpL}$, retinas from the 6x groups showed continued signs of Müller cell

287 disorganization. GFAP immunohistochemistry revealed obvious disorganization of GFAP within

288 the Müller glia with evidence of redistribution of the GFAP fibers away from the endfeet and

289 towards the cell body, perhaps indicative of a hypertrophy phenotype (Fig. 4A-C). These data

290 suggest that $6 x$ rounds of light damage resulted in increased signs of Müller glia stress and

291 gliosis, but did not affect their ability to re-enter the cell cycle and produce retinal progenitors.

292 Next, we analyzed the expression of genes that have been associated with proliferation in

293 other cellular events, such as cyclin dependent kinase 1 (cdk1), and c-jun (Malumbres 2014;

294 Munshi \& Ramesh 2013), and genes shown to be required for amplification of the progenitor 
295 population during retinal regeneration, such as proliferating cell nuclear antigen ( $p c n a)$, paired 296 box protein 6 (pax6a and pax6b), sonic hedgehog (shha and shhb), sinus oculis homeobox 3

297 (six3b), and transforming growth factor-beta-induced factor 1 (tgifl) (Fuccillo et al. 2006;

298 Hatton et al. 2006; Kaur et al. 2018; Lenkowski et al. 2013; Sherpa et al. 2014; Thomas et al. 299 2018; Thummel et al. 2010; Thummel et al. 2008b; Todd \& Fischer 2015). Compared with 1x 300 control retinas, retinas that underwent $6 \mathrm{x}$ rounds of light damage exhibited a significant 301 downregulation of many pro-proliferative genes, including $c d k 1, p c n a$, shhb, six $3 b$, and tgifl 302 (Fig. 4J). However, no change was observed for c-jun and pax6a, and one pro-proliferative gene, 303 shha, was significantly upregulated (Fig. 4J). In addition, we analyzed a group of genes 304 associated with Müller glial function of retinal homeostasis and support, such as glutamine 305 synthetase (glulb), potassium inward-rectifying channel (kcnj10a), and retinaldehyde binding 306 protein 1 (rlbp 1b) (Bringmann \& Wiedemann 2012). Compared with 1x control retinas, 6x 307 retinas exhibited a significant downregulation of $g l u l B$ and $r l b p 1 b$ (Fig. 4J), suggesting that the 308 Müller glia have begun to lose their ability to recycle extracellular glutamate and participate in 309 the regeneration of the cone visual pigment. Notably, expression of rlbpla, which is expressed in 310 the retinal pigmented epithelium but not in Müller glia (Collery et al. 2008), was not 311 significantly different between groups (Fig. 4J). Finally, we analyzed the expression of two 312 intermediate filaments of the Müller glia, gfap and vimentin (vim), and found no differences in 313 gfap expression and a significant downregulation of vim expression between groups at $72 \mathrm{hpL}$

314 (Fig. 4J). Together, these data suggested a complex set of consequences to 6x rounds of light 315 treatment in regards to maintaining proliferation of the progenitor pool and Müller glia function. 
317 Multiple rounds of light damage results in aberrant long-term regeneration of the inner

318 retina, a reduction of regenerated long single cones, and full regeneration of all other

319 photoreceptor subtypes.

320 Finally, we analyzed 1x and 6x groups at $28 \mathrm{dpL}$, an established time-point for

321 phenotypically defined full regeneration as previously described (Thomas 2015; Thummel et al.

322 2008a; Thummel et al. 2008b). Immunolocalization of GFAP showed an apparent resolution in

323 Müller cell gliosis and organization at $28 \mathrm{dpL}$ in both $1 \mathrm{x}$ and $6 \mathrm{x}$ retinas (Fig. 5A-C).

324 Interestingly, immunolocalization of $\mathrm{HuC} / \mathrm{D}$, a marker of amacrine and ganglion cells,

325 demonstrated aberrant localization of $\mathrm{HuC} / \mathrm{D}$-positive cells in both the $1 \mathrm{x}$ and $6 \mathrm{x}$ retinas

326 compared with non-light treated control retinas (Fig. 5D-F). In addition, we observed a

327 significantly greater number of $\mathrm{HuC} / \mathrm{D}$-positive cells in the inner plexiform layer (Fig. 5F,G

328 arrowhead; $p<0.05$ ) and outer retinal layer (Fig. 5F, G; arrow; $p<0.05$ ) in the 6x retinas

329 compared with the $1 \mathrm{x}$ retinas. This is consistent with reports demonstrating that Müller glial cells

330 can produce excess neurons even after one round of regeneration (Hitchcock \& Raymond 1992;

331 Powell et al. 2016; Sherpa et al. 2008; Sherpa et al. 2014).

332 Lastly, we compared photoreceptor numbers in untreated control retinas with 1x and 6x

333 retinas at $28 \mathrm{dpL}$ by immunolabeling the rod and cone photoreceptor populations with cell-

334 specific antibodies. Immunolocalization of rhodopsin (rods), Zpr-1 (red-green double cones),

335 blue opsin (long single cones), or UV opsin (short single cones) showed that retinas subjected to

$3361 \mathrm{x}$ and $6 \mathrm{x}$ rounds of light treatment regenerated both rods and cones to levels observed in

337 undamaged control retinas (Fig. 6). In contrast, blue cones exhibited reduced regeneration in the

$3386 x$ retinas, but not the $1 x($ Fig. $6 \mathrm{O}, \mathrm{p}<0.02)$ and UV cones regenerated in elevated numbers in the

$3391 \mathrm{x}$, but not the $6 \mathrm{x}$ retinas (Fig. 6P, p<0.03). These data indicated that although six rounds of light 
340 damage results in a significant increase in the acute gliotic response to damage, the Müller glia

341 largely retained their innate ability to regenerate photoreceptors following multiple rounds of

342 phototoxic lesion.

343

344 
Our first hypothesis of this study predicted that upon subsequent rounds of phototoxic

347 lesion, the exact same subset of Müller glia will not re-enter the cell cycle. We demonstrated that

$34835 \%$ of Müller glia re-entered the cell cycle in response to both phototoxic lesions, $24 \%$ of

349 Müller glia re-entered the cell cycle only during the first round of light damage, and $40 \%$ of

350 Müller glia re-entered the cell cycle only during the second round of phototoxic lesion (Fig 1).

351 This was indicated by the presence of GFAP-positive cells which were single-immunolabeled

352 with either anti-BrdU or anti-PCNA following the second round of phototoxic lesion (Fig 1D,

353 arrowheads and arrows, respectively). These data suggest that the pool of Müller glia is

354 heterogeneous, with a subset of Müller glia responding to the first round of lesion, and a

355 different, but overlapping subset responding to the second lesion. It is possible that this serves to

356 randomize the population of Müller glia that will respond to any given level of damage.

357 Our second aim of this study was to determine if there was an increase in the gliotic

358 response of the Müller glia with additional rounds of phototoxic treatment. Zebrafish Müller glial

359 cells undergo a reactive gliosis response at $18 \mathrm{hpL}$, which is characterized by cellular

360 hypertrophy in the outer nuclear layer (ONL) and physical wrapping of existing photoreceptor

361 nuclei (Thomas 2015). This gliotic phenomenon persists at $36 \mathrm{hpL}$, when $\sim 50 \%$ of zebrafish

362 Müller glial cells re-enter the cell cycle (Thomas 2015; Thummel et al. 2008b), but is then down-

363 regulated as progenitor amplification reaches its peak at $72 \mathrm{hpL}$ (Thummel et al. 2008a). Thus,

364 similar to what is observed during zebrafish cardiac regeneration, the onset of proliferation

365 occurs in a gliotic/fibrotic environment and only after a large number of progenitors are present

366 does the tissue downregulate this reactive state (Gonzalez-Rosa et al. 2011; Schnabel et al.

367 2011). Here we show that retinas that underwent $6 x$ rounds of light treatment exhibited a gliotic 
368 response that was phenotypically distinct from retinas that underwent 1x round of damage. First,

369 there was a significant upregulation of $g$ fap expression in $6 \mathrm{x}$ retinas at $36 \mathrm{hpL}$ (Fig. 3K). In

370 addition, there was an obvious dysmorphology in the 6x Müller glia at both 36 and $72 \mathrm{hpL}$ as

371 indicated by GFAP and Glutamine Synthetase immunolabelling (Figs. 3 and 4). Specifically,

372 GFAP immunolocalization appears to shift from being largely restricted to apical and basal

373 endfeet of Müller glia in untreated control retinas, to increasingly more centralized expression in

374 thick, disorganized fibers in the $1 \mathrm{x}$ and $6 \mathrm{x}$ retinas (Fig.4A-C). Since $g$ fap $\mathrm{mRNA}$ expression in

$3756 \mathrm{x}$ retinas at $72 \mathrm{hpL}$ is comparable to $1 \mathrm{x}$ retinas (Fig. $4 \mathrm{~J}$ ), this shift in immunlocalization may

376 represent a redistribution of existing GFAP filaments needed to support hypertrophied cells.

377 We also observed an upregulation in stat $3 \mathrm{mRNA}$ expression at $36 \mathrm{hpL}$ in $6 \mathrm{X}$ retinas

378 (Fig. 3K), which is indicative of an inflammatory stress response (Gorsuch \& Hyde 2014;

379 Nicolas et al. 2013), and may be associated with the increased numbers of 4C4-positive

$380 \mathrm{microglia} / \mathrm{macrophages}$ at this time point (Fig. 2P). Microglia are known to release pro-

381 inflammatory factors such as IL-6 that activate Stat3 in response to CNS damage (reviewed in

382 (Smith et al. 2012)). 4C4-positive cells, which are resident and distributed throughout

383 undamaged retinas, have previously been shown to proliferate and migrate from their existing

384 locations to an area of retinal damage (Craig et al. 2008; Raymond et al. 2006; Wu et al. 2001).

385 At this point it is unclear as to whether the increased number of 4C4-positive cells observed in

386 the $6 x$ retinas were a result of increased proliferation of existing microglia/macrophages or

387 whether following each additional round of phototoxic lesion the population of resident 4C4-

388 positive microglia/macrophages increased and remained in the retina.

A previous report comparing different intensities of phototoxic lesions demonstrated that

390 the greater the amount of photoreceptor loss, the more Müller glia re-enter the cell cycle 
391 (Thomas et al. 2012). Interestingly, here we observed that while photoreceptor loss was more

392 severe at $36 \mathrm{hpL}$ in retinas that underwent $6 \mathrm{x}$ rounds of light treatment, the proliferation response

393 did not appear to be subsequently increased in these retinas, as indicated by similar numbers of

394 PCNA-positive Müller glia in both the $1 \mathrm{x}$ and 6x retinas (Fig. 3B-C, J). These data could suggest

395 that there is a ceiling with respect to how much cell proliferation can be included by light

396 damage. However, we also observed a complex re-patterning of cell signaling programs in

397 Müller glia responding to retinas that have been exposed to multiple rounds of phototoxic lesion

398 compared to those that have only undergone one round of damage. For example, we observed a

399 downregulation of many pro-proliferative genes in $6 x$ retinas at $72 \mathrm{hpL}$, including $c d k 1, p c n a$,

400 and $\operatorname{shhb}$ (Fig. 4J), and additional signs of impaired Müller glial cell function (vim, glulB, rlbpB)

401 at $72 \mathrm{hpL}$ in $6 \mathrm{x}$ retinas compared to $1 \mathrm{x}$ (Fig. $4 \mathrm{~J})$.

402 In addition to these early abnormalities, we also observed aberrant localization of inner

403 retinal neurons in the inner plexiform layer (IPL) and in the outer retina at $28 \mathrm{dpL}$ in retinas that

404 underwent 6 rounds of light damage (Fig. 5D-F). Because BrdU labeling was not performed

405 throughout these experiments, we cannot determine when they arose or whether they arose from

406 aberrant differentiation of retinal progenitors or from aberrant migration of existing inner retinal

407 neurons. However, these data are consistent with a recent report comparing multiple retinal

408 damage paradigms that found that Müller glia regenerate excess neurons during the regenerative

409 process (Powell et al. 2016). Indeed, we note that there appears to be a trend of slow build-up of

410 these aberrantly localized retinal neurons with increasing amount of phototoxic lesion when you

411 compare the 0,1 , and $6 \mathrm{x}$ retinas (Fig. 5G). Thus, while it is likely that the extent of cell mis-

412 localization gradually builds with each damage event, additional studies are required to validate

413 this hypothesis. This is an interesting finding because inner retinal neurons are not damaged by 
414 the light lesion, so signals to regenerate inner retinal neurons should not be present. However, we

415 noted a significant upregulation of shha at the $72 \mathrm{hpL}$ time point (Fig. 4J). In addition to being

416 associated with late proliferation of the retinal progenitors, Shha is required for the development

417 of amacrine and ganglion cells (Neumann \& Nuesslein-Volhard 2000). Thus, an excess of Shha

418 signaling at this time point may erroneously drive a subset of the progenitors toward an amacrine

419 or ganglion cell fate.

420 We terminated the study following 6 rounds of light treatment and 28 day recovery

421 period so as not to exceed the normal age window of 9-18 months that we use for light lesion

422 studies. However, we acknowledge that the age of the $6 \mathrm{x}$ treated fish (18mo vs. 9mo in $1 \mathrm{x}$ fish)

423 may be a confounding factor in our findings, including gene expression, gliosis, and aberrancy in

424 regeneration. Indeed, to our knowledge, age has never been directly tested as a confounding

425 factor in retinal regeneration studies. Therefore, further studies are ongoing in our lab to

426 investigate the aging Müller glia response to retinal damage and to clearly define for the field a

427 window of adulthood within which age is not a factor in retinal regeneration. In other tissues,

428 such as the zebrafish telencephalon, it has been reported that the regenerative capacity of the

429 radial glia, a comparable cell type to the Müller glia, declines with advanced age (Edelmann et

430 al. 2013). This group noted that there is a decrease in the number of dividing maintenance radial

431 glia with a concurrent increase in cell cycle time in $18 \mathrm{mo}$ old fish as compared to $4.5 \mathrm{mo}$ old fish

432 at baseline. In response to injury, fewer radial glia re-entered the cell cycle to initiate

433 regeneration in $28 \mathrm{mo}$ old fish compared to $8 \mathrm{mo}$ old fish (Edelmann et al. 2013). These

434 experiments could indicate that the stem cell populations of the CNS appear to exhaust with age.

435 In contrast, we observed no significant difference in the percentage of Müller glia cells that re-

436 entered the cell cycle between the 1x (9mo) and 6x (18mo) groups (Fig. 3J). Regarding the 
437 inflammatory response to regeneration, it was previously shown that aged zebrafish fail to recruit

438 4C4-positive microglia/macrophages to the site of a demyelinating injury of the optic nerve and

439 that the quality of subsequent re-myelination is significantly reduced in aged animals (Munzel et

440 al. 2014). In our experiments, the 6x treatment group shows a significantly higher number of

441 4C4-positive cells in the retina. However, this finding could be a result of a slow build-up of

442 4C4-positive cells following each round of damage and does not rule out the possibility that

443 fewer and fewer 4C4-positive cells are recruited with each subsequent round.

444 Despite the increase in gliosis and aberrancies seen in recovery after phototoxic lesion,

445 we determined that Müller glia retain the ability to re-enter the cell cycle and regenerate

446 photoreceptors following multiple rounds of phototoxic lesion. The increased acute gliotic

447 response of the Müller glia in retinas that were exposed to multiple rounds of phototoxic lesion

448 may indeed suggest that they are undergoing remodeling following each round of damage and

449 thus take on a different response phenotype as the rounds of damage increase. However, our

450 findings highlight the incredible capacity of the Müller glia to participate in the regenerative

451 response even in a gliotic state. In addition, to our knowledge, this is the first description of the

452 response of the zebrafish retinal Müller glia to multiple rounds of phototoxic lesion. These

453 findings open new avenues for future investigation into genetic programs that alter the function

454 of the Müller cell gliotic response, and perhaps, elucidate parallels in the mammalian system that

455 help us to better understand the delicate balance between gliosis and stemness of this important 456 retinal cell population.

457

458 
459

460

461

462

463

464

465

466

467

468

469

470

471

472

473

474

475

476

477

478

479

480

481

482

\section{ACKNOWLEDGEMENTS}

The authors would like to thank Xixia Luo for excellent fish husbandry and technical support. 
Figure 1. Following consecutive rounds of light damage, different populations of Müller

486

487

488

489

490

491

492

493

494

495

496

497

498

499

500

501

502

503

504

505

glia re-enter the cell cycle. A) Timeline of the BrdU and light lesion, fish were exposed to two rounds of light lesion separated by 28 day period of standard light/dark cycling. B-D) Retinal sections collected at $36 \mathrm{hpL}$ immunolabeled with anti-GFAP (blue), anti-BrdU (green), antiPCNA (red) and DAPI (nuclear stain; grey). Arrowheads point to BrdU-positive cells, arrows

point to PCNA-positive cells, and double arrowheads point to cells co-immunolabeled with both PCNA and BrdU. E) An inset of image D. F) Quantification of the average numbers of BrdUpositive cells (24\%), PCNA positive cells (40\%) and co-labeled cells (35\%) counted over a linear distance of $300 \mu \mathrm{m}$ on the central dorsal retina following two consecutive rounds of light damage. Scale bar represents $25 \mu \mathrm{m}$ in B and $12 \mu \mathrm{m}$ in E.

Figure 2. Multiple rounds of light treatment leads to greater photoreceptor loss at 36 hours of light treatment. A) Experimental design used for multiple rounds of light treatment. B-P)

Retinal sections collected at $36 \mathrm{hpL}$ immunolabeled with anti-rhodopsin, Zpr-1, anti-blue opsin, anti-UV opsin, and 4C4 to show the varying amounts of damage to photoreceptors in untreated control (No Light Treatment), experimental control (1x Light Treatment), and experimental retinas (6x Light Treatment). Nuclei are stained blue with TO-PRO-3. B-D) Rod photoreceptor outer segments are immunolabeled with anti-rhodopsin (red). E-G) Red-green double cones are immunolabeled with Zpr-1 (green). H-J) Long single cones are immunolabeled with anti-blue opsin (green). K-M) Short single cones are immunolabeled with anti-UV opsin (green). N-P) Microglia/macrophages are immunolabeled with 4C4 (red). In the images taken after six rounds 
506 of light treatment, arrows point to individual cone opsins in the large debris field. Q-T)

507 Quantification of average numbers of photoreceptors in untreated (0h), control (1x) and

508 experimental (6x) groups ( $n=5$ per group) counted over a linear distance of $300 \mu m$ on the central

509 dorsal retina. Asterisks indicates significant differences between groups ( 4 asterisks $\mathrm{p}<0.0001 ; 3$

510 asterisks $\mathrm{p}<0.0003 ; 2$ asterisks $\mathrm{p}<0.006 ; 1$ asterisk $\mathrm{p}<0.02$ ). Scale bar represents $25 \mu \mathrm{m}$.

511

512 Figure 3. Following six rounds of light treatment Müller glia become highly unorganized

513 and exhibit gliotic characteristics. A-C) Retinal sections collected at $36 \mathrm{hpL}$ immunolabeled

514 with anti-GFAP (red), and anti-PCNA (green) in untreated control (No Light Treatment),

515 experimental control (1x Light Treatment), and experimental retinas (6x Light Treatment). D-F)

516 Retinal sections collected at $36 \mathrm{hpL}$ immunolabeled with anti-glutamine synthetase (G.S.; green)

517 and TO-PRO-3 as nuclear stain (blue). G-I) Magnified inset of regions indicated by red box in

518 panels D-F. J) Quantification of average number of PCNA positive Müller glia in experimental

519 control and experimental groups $(n=5)$. Cells were counted over a linear distance of $300 \mu \mathrm{m}$ on

520 the central dorsal retina. K) Graph showing the average fold-change in expression of genes

521 associated with proliferation ( $p c n a$ ) and gliosis/stress (stat3, gfap) as determined by real-time

522 qPCR. Asterisk indicates significantly different from control $(\mathrm{p}<0.05)$. Scale bar represents $52325 \mu \mathrm{m}$.

524

525 Figure 4. Following six rounds of light treatment Müller glia remain persistently gliotic at

526 the canonical peak stage of progenitor proliferation and migration. A-I) Retinal sections

527 collected at $72 \mathrm{hpL}$ immunolabeled with anti-GFAP (red) and anti-PCNA (green) in untreated

528 control (No Light Treatment), experimental control (1x Light Treatment), and experimental 
529 retinas (6x Light Treatment). A-C) Müller glia are immunolabeled with anti-GFAP. D-F) Müller

530 glia that have re-entered the cell cycle are immunolabeled with anti-PCNA. G-I) Merge of anti-

531 GFAP and anti-PCNA immunolabeling. J) Graph showing the average fold-change in expression

532 of genes associated with (1) general proliferation (cdkl, c-jun), (2) retinal regeneration (shha,

533 shhb, pcna, pax6a, pax6b, six3b and tgifl), (3) Müller cell function ( glulb, gfap, kcnj10a,

$534 r l b p 1 b$, vim), and (4) RPE function ( $r l b p l a$ ). Asterisk indicates significantly different from

535 control $(\mathrm{p}<0.05)$. Scale bar represents $25 \mu \mathrm{m}$.

536

537 Figure 5. Following multiple rounds of regeneration, retinas exhibit abnormal localization

538 of inner retinal neurons. A-F) Retinal sections collected at $28 \mathrm{dpL}$ immunolabeled with anti-

539 GFAP and anti-HuC/D to show Müller glia and inner retinal neurons, respectively, in untreated

540 control (No Light Treatment), experimental control (1x Light Treatment) and experimental

541 retinas (6x Light Treatment). Nuclei are stained in blue with TO-PRO-3. A-C) Müller glia are

542 immunolabeled with anti-GFAP. D-F) Amacrine and ganglion cells are immunolabeled with

543 anti-HuC/D. Mis-localization of HuC/D-positive cells were observed in the inner plexiform layer

544 (IPL; arrowhead) and outer retina (arrow). G) Quantification of average numbers of HuC/D-

545 positive cells found in the IPL and outer retina ( $\mathrm{n}=5$ per group). Cells were counted over a linear

546 distance of $300 \mu \mathrm{m}$ on the central dorsal retina. Asterisk indicates significantly different from $0 \mathrm{hr}$

547 control; Psi symbol indicates significantly different from $1 \mathrm{x}$ retinas $(\mathrm{p}<0.05)$. Scale bar

548 represents $25 \mu \mathrm{m}$.

549 
551 Figure 6. Following six rounds of light treatment, retinas replace the lost photoreceptors.

552 A-L) Retinal sections collected at 28 dpL immunolabeled with anti-rhodopsin, Zpr-1, anti-blue 553 opsin, and anti-UV opsin to show regeneration of the photoreceptors in untreated control (No 554 Light Treatment), experimental control (1x Light Treatment), and experimental retinas (6x Light 555 Treatment) following photolytic damage. S Nuclei are stained blue with TO-PRO-3. A-C). Rod 556 photoreceptor outer segments are immunolabeled with anti-rhodopsin (red). D-F) Red-green 557 double cones are immunolabeled with Zpr-1 (green). G-I) Long single cones are immunolabeled 558 with anti-blue opsin (green). J-L) Short single cones are immunolabeled with anti-UV opsin 559 (green). M-P) Quantification of the average number of photoreceptors $28 \mathrm{dpL}$ in control and 560 experimental groups $(n=5)$. Cells were counted over a linear distance of $300 \mu m$ on the central 561 dorsal retina. Single asterisks indicate significant differences between groups $(\mathrm{p}<0.03)$. Scale bar 562 represents $25 \mu \mathrm{m}$.

563

564 Supplemental Figure 1. Comparison of photoreceptor degeneration and GFAP 565 expression at 36 hpL between 9 and 18 mo zebrafish. A-B) Retinal sections from 9 mo

566 (A) and 18 mo (B) zebrafish immunolabeled with GFAP (Müller glia; green), Zpr-3 (Rod

567 photoreceptors; red), and the nuclear stain TO-PRO-3 (blue). No differences in Müller glial 568 reactivity or photoreceptor degeneration was observed. Scale bar represents $25 \mu \mathrm{m}$. 
Azevedo AS, Grotek B, Jacinto A, Weidinger G, and Saude L. 2011. The regenerative capacity of the zebrafish caudal fin is not affected by repeated amputations. PLoS One 6:e22820.

Bringmann A, Pannicke T, Grosche J, Francke M, Wiedemann P, Skatchkov SN, Osborne NN, and Reichenbach A. 2006. Muller cells in the healthy and diseased retina. Prog Retin Eye Res 25:397-424.

Bringmann A, and Wiedemann P. 2012. Muller glial cells in retinal disease. Ophthalmologica 227:1-19.

Chablais F, Veit J, Rainer G, and Jazwinska A. 2011. The zebrafish heart regenerates after cryoinjury-induced myocardial infarction. BMC Dev Biol 11:21.

Collery R, McLoughlin S, Vendrell V, Finnegan J, Crabb JW, Saari JC, and Kennedy BN. 2008. Duplication and divergence of zebrafish CRALBP genes uncovers novel role for RPEand Muller-CRALBP in cone vision. Invest Ophthalmol Vis Sci 49:3812-3820.

Craig SE, Calinescu AA, and Hitchcock PF. 2008. Identification of the molecular signatures integral to regenerating photoreceptors in the retina of the zebra fish.J Ocul Biol Dis Infor 1:73-84.

Edelmann K, Glashauser L, Sprungala S, Hesl B, Fritschle M, Ninkovic J, Godinho L, and Chapouton P. 2013. Increased radial glia quiescence, decreased reactivation upon injury and unaltered neuroblast behavior underlie decreased neurogenesis in the aging zebrafish telencephalon. J Comp Neurol 521:3099-3115.

Fuccillo M, Joyner AL, and Fishell G. 2006. Morphogen to mitogen: the multiple roles of hedgehog signalling in vertebrate neural development. Nat Rev Neurosci 7:772-783.

Gonzalez-Rosa JM, Martin V, Peralta M, Torres M, and Mercader N. 2011. Extensive scar formation and regression during heart regeneration after cryoinjury in zebrafish. Development 138:1663-1674.

Gorsuch RA, and Hyde DR. 2014. Regulation of Muller glial dependent neuronal regeneration in the damaged adult zebrafish retina. Exp Eye Res 123:131-140.

Hatton BA, Knoepfler PS, Kenney AM, Rowitch DH, de Alboran IM, Olson JM, and Eisenman RN. 2006. N-myc is an essential downstream effector of Shh signaling during both normal and neoplastic cerebellar growth. Cancer Res 66:8655-8661.

Hitchcock PF, and Raymond PA. 1992. Retinal regeneration. Trends Neurosci 15:103-108. Kassen SC, Ramanan V, Montgomery JE, C TB, Liu CG, Vihtelic TS, and Hyde DR. 2007. Time course analysis of gene expression during light-induced photoreceptor cell death and regeneration in albino zebrafish. Dev Neurobiol 67:1009-1031.

Kassen SC, Thummel R, Burket CT, Campochiaro LA, Harding MJ, and Hyde DR. 2008. The $\mathrm{Tg}$ (ccnb1:EGFP) transgenic zebrafish line labels proliferating cells during retinal development and regeneration. Mol Vis 14:951-963.

Kaur S, Gupta S, Chaudhary M, Khursheed MA, Mitra S, Kurup AJ, and Ramachandran R. 2018. let-7 MicroRNA-Mediated Regulation of Shh Signaling and the Gene Regulatory Network Is Essential for Retina Regeneration. Cell Rep 23:1409-1423.

Lenkowski JR, Qin Z, Sifuentes CJ, Thummel R, Soto CM, Moens CB, and Raymond PA. 2013. Retinal regeneration in adult zebrafish requires regulation of TGFbeta signaling. Glia 61:1687-1697. 
617 Lenkowski JR, and Raymond PA. 2014. Muller glia: Stem cells for generation and

618

619

620

621

622

623

624

625

626

627

628

629

630

631

632

633

634

635

636

637

638

639

640

641

642

643

644

645

646

647

648

649

650

651

652

653

654

655

656

657

658

659

660

661

662

regeneration of retinal neurons in teleost fish. Prog Retin Eye Res 40:94-123.

Malumbres M. 2014. Cyclin-dependent kinases. Genome Biol 15:122.

Munshi A, and Ramesh R. 2013. Mitogen-activated protein kinases and their role in radiation response. Genes Cancer 4:401-408.

Munzel EJ, Becker CG, Becker T, and Williams A. 2014. Zebrafish regenerate full thickness optic nerve myelin after demyelination, but this fails with increasing age. Acta Neuropathol Commun 2:77.

Neumann CJ, and Nuesslein-Volhard C. 2000. Patterning of the zebrafish retina by a wave of sonic hedgehog activity. Science 289:2137-2139.

Nicolas CS, Amici M, Bortolotto ZA, Doherty A, Csaba Z, Fafouri A, Dournaud P, Gressens P, Collingridge GL, and Peineau S. 2013. The role of JAK-STAT signaling within the CNS. JAKSTAT 2:e22925.

Pfaffl MW. 2001. A new mathematical model for relative quantification in real-time RT-PCR. Nucleic Acids Res 29:e45.

Poss KD, Wilson LG, and Keating MT. 2002. Heart regeneration in zebrafish. Science 298:2188-2190.

Powell C, Cornblath E, Elsaeidi F, Wan J, and Goldman D. 2016. Zebrafish Muller gliaderived progenitors are multipotent, exhibit proliferative biases and regenerate excess neurons. Sci Rep 6:24851.

Qin Z, Barthel LK, and Raymond PA. 2009. Genetic evidence for shared mechanisms of epimorphic regeneration in zebrafish. Proc Natl Acad Sci U S A 106:9310-9315.

Raymond PA, Barthel LK, Bernardos RL, and Perkowski JJ. 2006. Molecular characterization of retinal stem cells and their niches in adult zebrafish. BMC Dev Biol 6:36.

Richardson R, Slanchev K, Kraus C, Knyphausen P, Eming S, and Hammerschmidt M. 2013. Adult zebrafish as a model system for cutaneous wound-healing research.J Invest Dermatol 133:1655-1665.

Schnabel K, Wu CC, Kurth T, and Weidinger G. 2011. Regeneration of cryoinjury induced necrotic heart lesions in zebrafish is associated with epicardial activation and cardiomyocyte proliferation. PLoS One 6:e18503.

Sherpa T, Fimbel SM, Mallory DE, Maaswinkel H, Spritzer SD, Sand JA, Li L, Hyde DR, and Stenkamp DL. 2008. Ganglion cell regeneration following whole-retina destruction in zebrafish. Dev Neurobiol 68:166-181.

Sherpa T, Lankford T, McGinn TE, Hunter SS, Frey RA, Sun C, Ryan M, Robison BD, and Stenkamp DL. 2014. Retinal regeneration is facilitated by the presence of surviving neurons. Dev Neurobiol 74:851-876.

Smith JA, Das A, Ray SK, and Banik NL. 2012. Role of pro-inflammatory cytokines released from microglia in neurodegenerative diseases. Brain Res Bull 87:10-20.

Thomas JL, Morgan GW, Dolinski KM, and Thummel R. 2018. Characterization of the pleiotropic roles of Sonic Hedgehog during retinal regeneration in adult zebrafish. Exp Eye Res 166:106-115.

Thomas JL, Nelson CM, Luo X, Hyde DR, and Thummel R. 2012. Characterization of multiple light damage paradigms reveals regional differences in photoreceptor loss. Exp Eye Res 97:105-116.

Thomas JLR, A. H.; Morgan, G. W.; Thummel, R. 2015. Reactive Gliosis in the Adult Zebrafish Retina. Exp Eye Res.

Peer] reviewing PDF | (2018:02:25857:2:0:NEW 14 Aug 2018) 
663 Thummel R, Enright JM, Kassen SC, Montgomery JE, Bailey TJ, and Hyde DR. 2010. Pax6a

664

665

666

667

668

669

670

671

672

673

674

675

676

677

678

679

680

681

682 and Pax6b are required at different points in neuronal progenitor cell proliferation during zebrafish photoreceptor regeneration. Exp Eye Res 90:572-582.

Thummel R, Kassen SC, Enright JM, Nelson CM, Montgomery JE, and Hyde DR. 2008a. Characterization of Muller glia and neuronal progenitors during adult zebrafish retinal regeneration. Exp Eye Res 87:433-444.

Thummel R, Kassen SC, Montgomery JE, Enright JM, and Hyde DR. 2008b. Inhibition of Muller glial cell division blocks regeneration of the light-damaged zebrafish retina. Dev Neurobiol 68:392-408.

Todd L, and Fischer AJ. 2015. Hedgehog signaling stimulates the formation of proliferating Muller glia-derived progenitor cells in the chick retina. Development 142:26102622.

Vihtelic TS, and Hyde DR. 2000. Light-induced rod and cone cell death and regeneration in the adult albino zebrafish (Danio rerio) retina. J Neurobiol 44:289-307.

Westerfield. 1995. The Zebrafish Book: A guide for the laboratory use of zebrafish (Danio rerio). Eugene, OR: Univ. of Oregon Press.

Wu DM, Schneiderman T, Burgett J, Gokhale P, Barthel L, and Raymond PA. 2001. Cones regenerate from retinal stem cells sequestered in the inner nuclear layer of adult goldfish retina. Invest Ophthalmol Vis Sci 42:2115-2124. 


\section{Figure 1}

Following consecutive rounds of light damage, different populations of Müller glia reenter the cell cycle.
A) Timeline of the BrdU and light lesion, fish were exposed to two rounds of light lesion separated by 28 day period of standard light/dark cycling. B-D) Retinal sections collected at $36 \mathrm{hpL}$ immunolabeled with anti-GFAP (blue), anti-BrdU (green), anti-PCNA (red) and DAPI (nuclear stain; grey). Arrowheads point to BrdU-positive cells, arrows point to PCNA-positive cells, and double arrowheads point to cells co-immunolabeled with both PCNA and BrdU. E) An inset of image D. F) Quantification of the average numbers of BrdU-positive cells (24\%), PCNA positive cells (40\%) and co-labeled cells (35\%) counted over a linear distance of $300 \mu \mathrm{m}$ on the central dorsal retina following two consecutive rounds of light damage. Scale bar represents $25 \mu \mathrm{m}$ in $\mathrm{B}$ and $12 \mu \mathrm{m}$ in $\mathrm{E}$. 

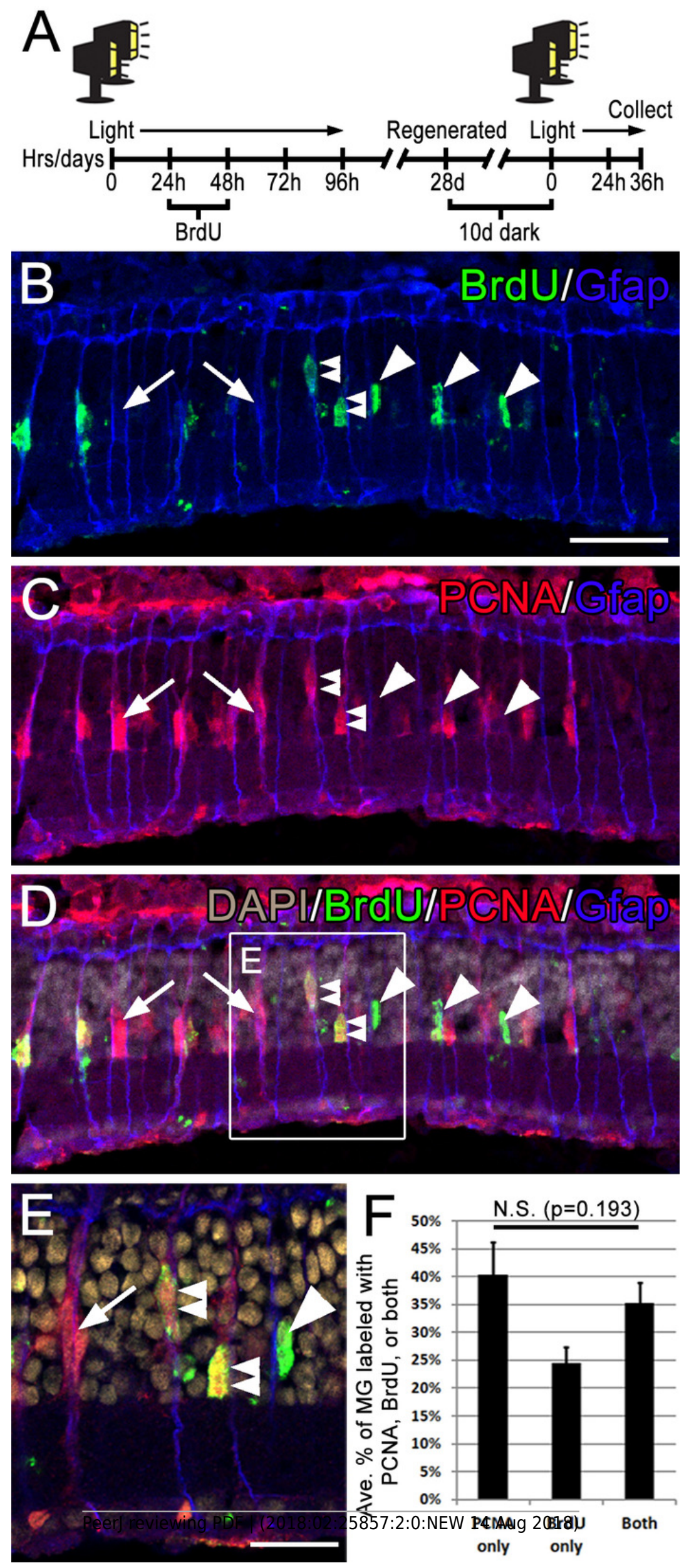


\section{Figure 2}

Multiple rounds of light treatment leads to greater photoreceptor loss at 36 hours of light treatment.

A) Experimental design used for multiple rounds of light treatment. B-P) Retinal sections collected at $36 \mathrm{hpL}$ immunolabeled with anti-rhodopsin, Zpr-1, anti-blue opsin, anti-UV opsin, and 4C4 to show the varying amounts of damage to photoreceptors in untreated control (No Light Treatment), experimental control (1x Light Treatment), and experimental retinas (6x Light Treatment). Nuclei are stained blue with TO-PRO-3. B-D) Rod photoreceptor outer segments are immunolabeled with anti-rhodopsin (red). E-G) Red-green double cones are immunolabeled with Zpr-1 (green). $\mathrm{H}-\mathrm{J}$ ) Long single cones are immunolabeled with anti-blue opsin (green). K-M) Short single cones are immunolabeled with anti-UV opsin (green). N-P) Microglia/macrophages are immunolabeled with 4C4 (red). In the images taken after six rounds of light treatment, arrows point to individual cone opsins in the large debris field. Q-T) Quantification of average numbers of photoreceptors in untreated (0h), control ( $1 \mathrm{x})$ and experimental (6x) groups ( $n=5$ per group) counted over a linear distance of $300 \mu m$ on the central dorsal retina. Asterisks indicates significant differences between groups ( 4 asterisks $p<0.0001 ; 3$ asterisks $p<0.0003 ; 2$ asterisks $p<0.006 ; 1$ asterisk $p<0.02$ ). Scale bar represents $25 \mu \mathrm{m}$. 

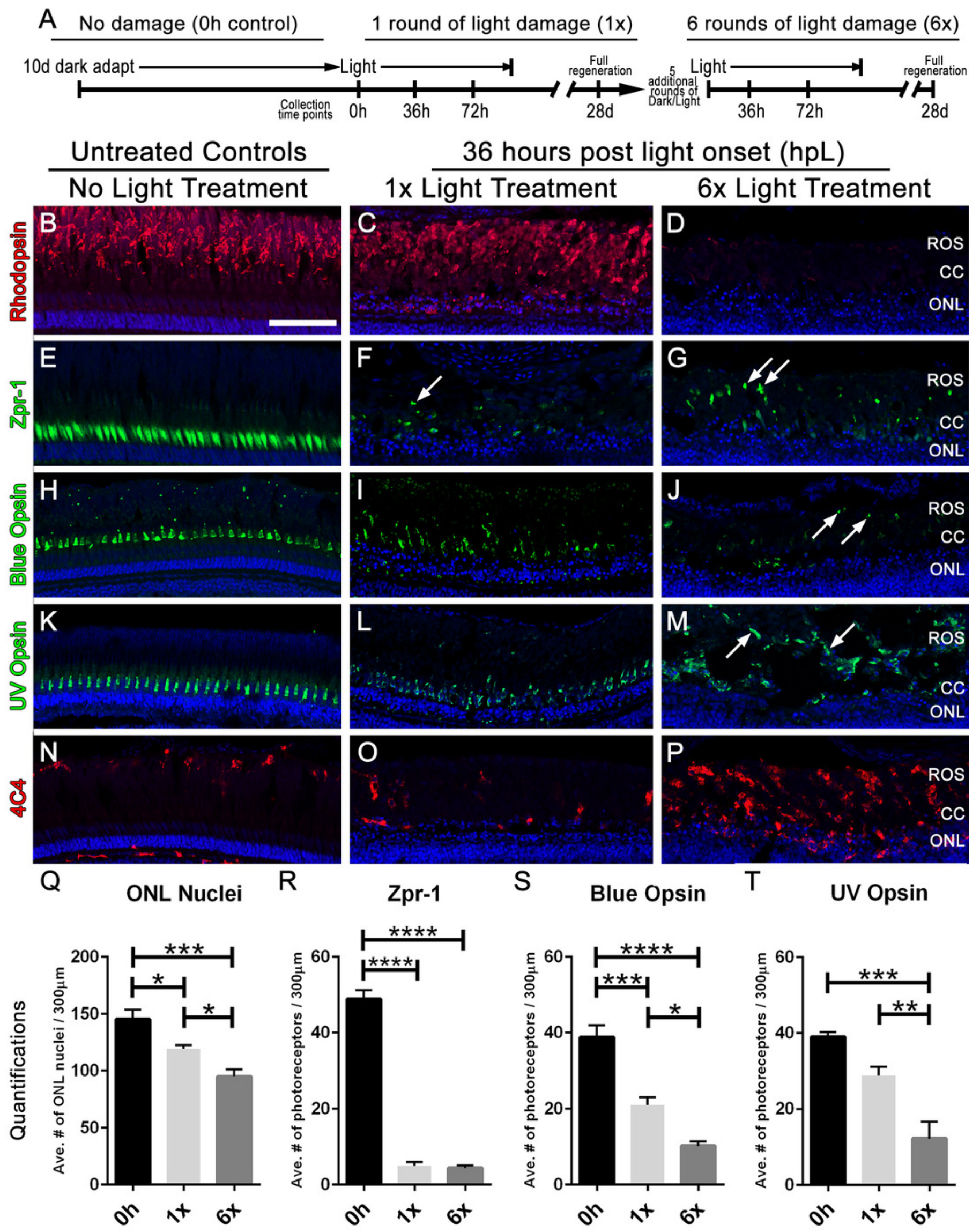


\section{Figure 3}

Following six rounds of light treatment Müller glia become highly unorganized and exhibit gliotic characteristics.

A-C) Retinal sections collected at $36 \mathrm{hpL}$ immunolabeled with anti-GFAP (red), and anti-PCNA (green) in untreated control (No Light Treatment), experimental control (1x Light Treatment), and experimental retinas (6x Light Treatment). D-F) Retinal sections collected at $36 \mathrm{hpL}$ immunolabeled with anti-glutamine synthetase (G.S.; green) and TO-PRO-3 as nuclear stain (blue). G-I) Magnified inset of regions indicated by red box in panels D-F. J) Quantification of average number of PCNA positive Müller glia in experimental control and experimental groups $(n=5)$. Cells were counted over a linear distance of $300 \mu \mathrm{m}$ on the central dorsal retina. K) Graph showing the average fold-change in expression of genes associated with proliferation (pcna) and gliosis/stress (stat3, gfap) as determined by real-time qPCR. Asterisk indicates significantly different from control $(p<0.05)$. Scale bar represents $25 \mu \mathrm{m}$. 


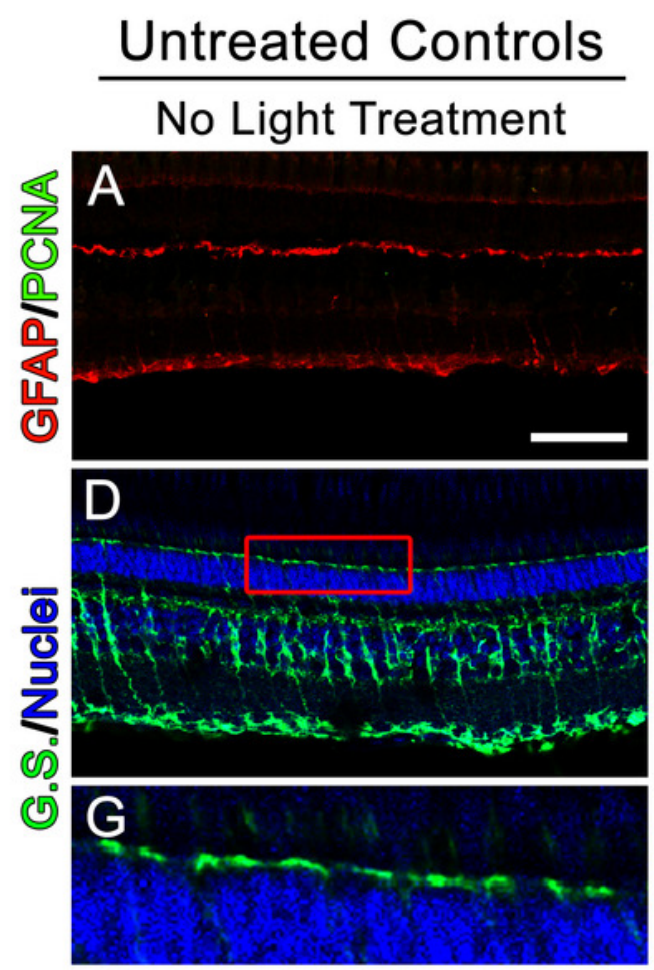

J PCNA+ Muller Glia

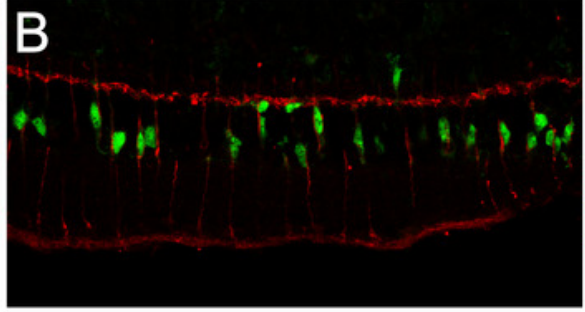

36 hours post light onset (hpL)

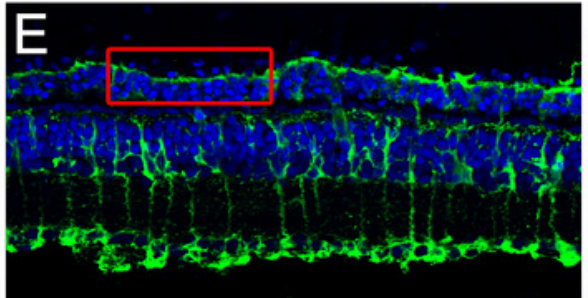

6x Light Treatment
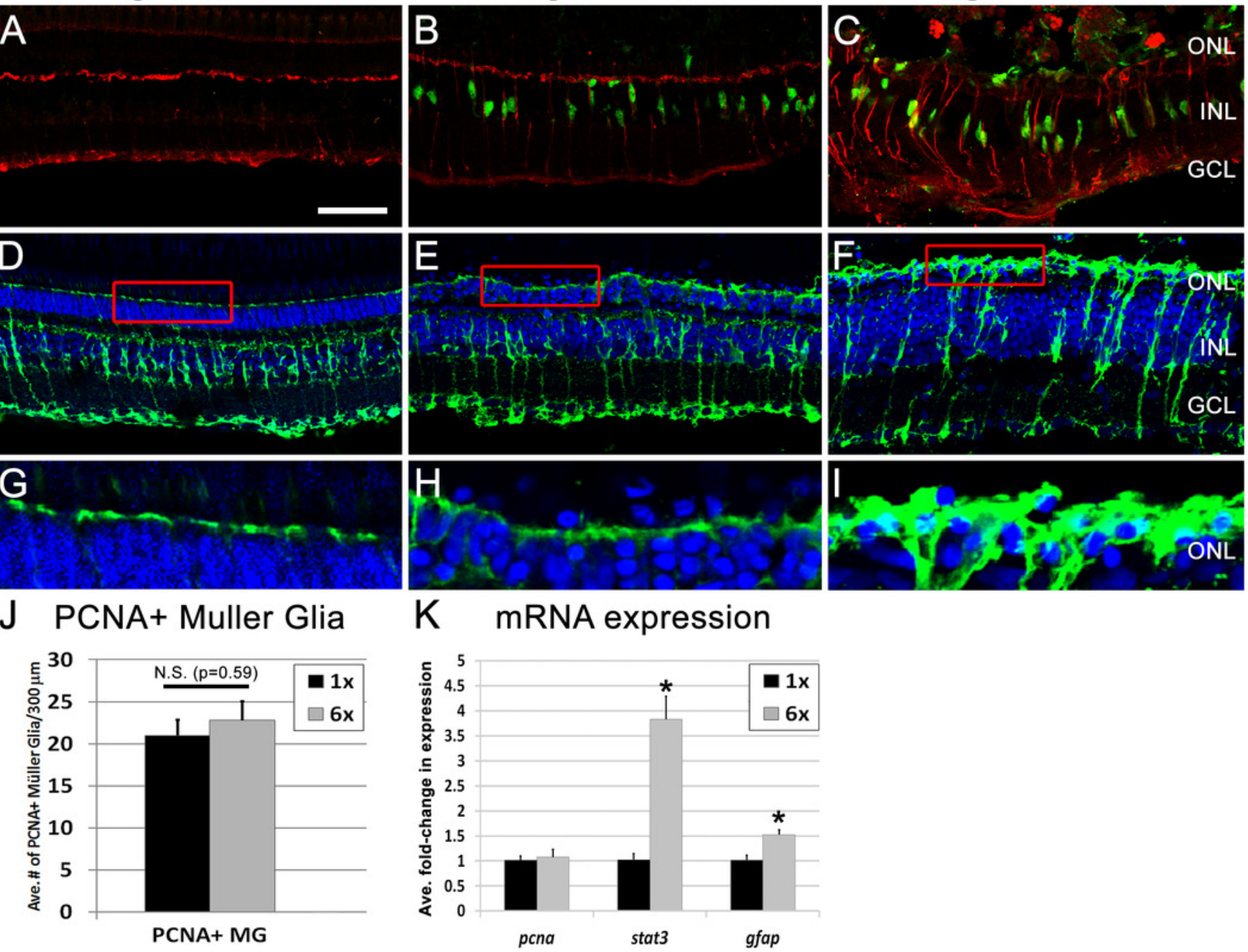

K mRNA expression

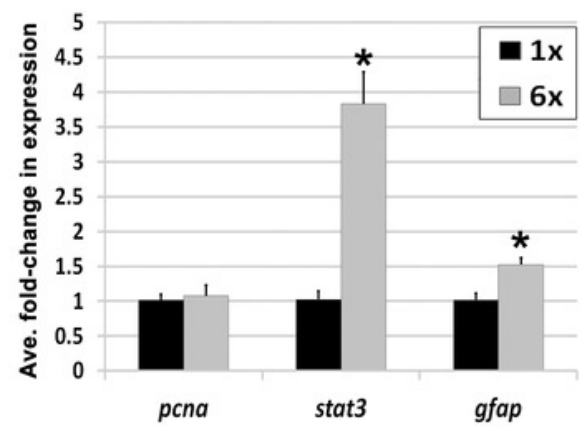




\section{Figure 4}

Following six rounds of light treatment Müller glia remain persistently gliotic at the canonical peak stage of progenitor proliferation and migration.

A-I) Retinal sections collected at $72 \mathrm{hpL}$ immunolabeled with anti-GFAP (red) and anti-PCNA (green) in untreated control (No Light Treatment), experimental control (1x Light Treatment), and experimental retinas (6x Light Treatment). A-C) Müller glia are immunolabeled with antiGFAP. D-F) Müller glia that have re-entered the cell cycle are immunolabeled with anti-PCNA. G-I) Merge of anti-GFAP and anti-PCNA immunolabeling. J) Graph showing the average foldchange in expression of genes associated with (1) general proliferation (cdk1, c-jun), (2) retinal regeneration (shha, shhb, pcna, pax6a, pax6b, six3b and tgif1), (3) Müller cell function ( glulb, gfap, kcnj10a, rlbp1b, vim), and (4) RPE function ( rlbpla ). Asterisk indicates significantly different from control $(p<0.05)$. Scale bar represents $25 \mu \mathrm{m}$. 


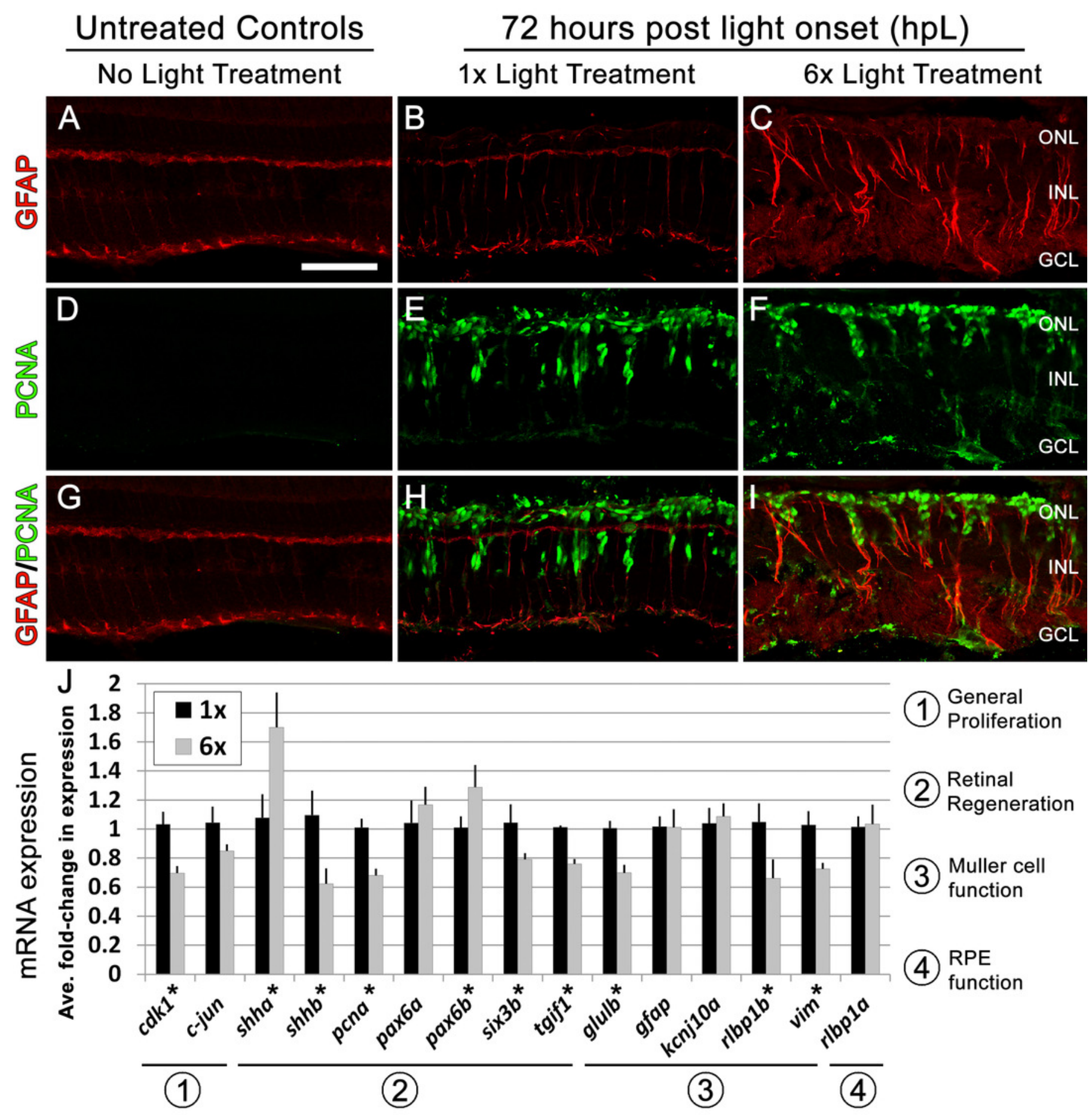




\section{Figure 5}

Following multiple rounds of regeneration, retinas exhibit abnormal localization of inner retinal neurons.

A-F) Retinal sections collected at $28 \mathrm{dpL}$ immunolabeled with anti-GFAP and anti-HuC/D to show Müller glia and inner retinal neurons, respectively, in untreated control (No Light Treatment), experimental control (1x Light Treatment) and experimental retinas (6x Light Treatment). Nuclei are stained in blue with TO-PRO-3. A-C) Müller glia are immunolabeled with anti-GFAP. D-F) Amacrine and ganglion cells are immunolabeled with anti-HuC/D. Mislocalization of HuC/D-positive cells were observed in the inner plexiform layer (IPL;

arrowhead) and outer retina (arrow). G) Quantification of average numbers of HuC/D-positive cells found in the IPL and outer retina ( $n=5$ per group). Cells were counted over a linear distance of $300 \mu \mathrm{m}$ on the central dorsal retina. Asterisk indicates significantly different from $0 \mathrm{hr}$ control; Psi symbol indicates significantly different from $1 \times$ retinas $(p<0.05)$. Scale bar represents $25 \mu \mathrm{m}$.

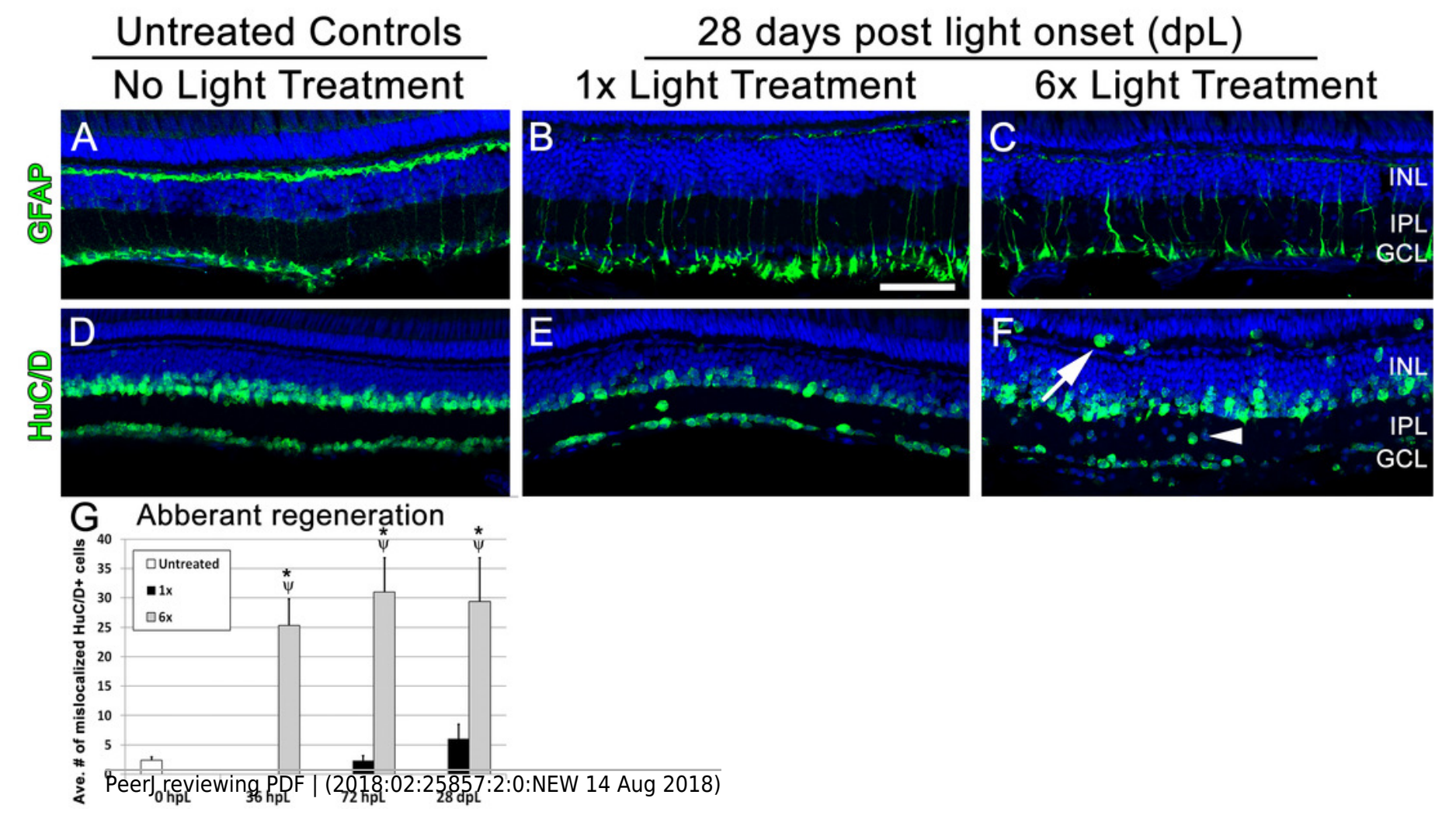




\section{Figure 6}

Following six rounds of light treatment, retinas replace the lost photoreceptors.

A-L) Retinal sections collected at $28 \mathrm{dpL}$ immunolabeled with anti-rhodopsin, Zpr-1, anti-blue opsin, and anti-UV opsin to show regeneration of the photoreceptors in untreated control (No Light Treatment), experimental control (1x Light Treatment), and experimental retinas (6x Light Treatment) following photolytic damage. S Nuclei are stained blue with TO-PRO-3. A-C). Rod photoreceptor outer segments are immunolabeled with anti-rhodopsin (red). D-F) Redgreen double cones are immunolabeled with Zpr-1 (green). G-I) Long single cones are immunolabeled with anti-blue opsin (green). J-L) Short single cones are immunolabeled with anti-UV opsin (green). M-P) Quantification of the average number of photoreceptors $28 \mathrm{dpL}$ in control and experimental groups $(n=5)$. Cells were counted over a linear distance of $300 \mu \mathrm{m}$ on the central dorsal retina. Single asterisks indicate significant differences between groups $(p<0.03)$. Scale bar represents $25 \mu \mathrm{m}$. 


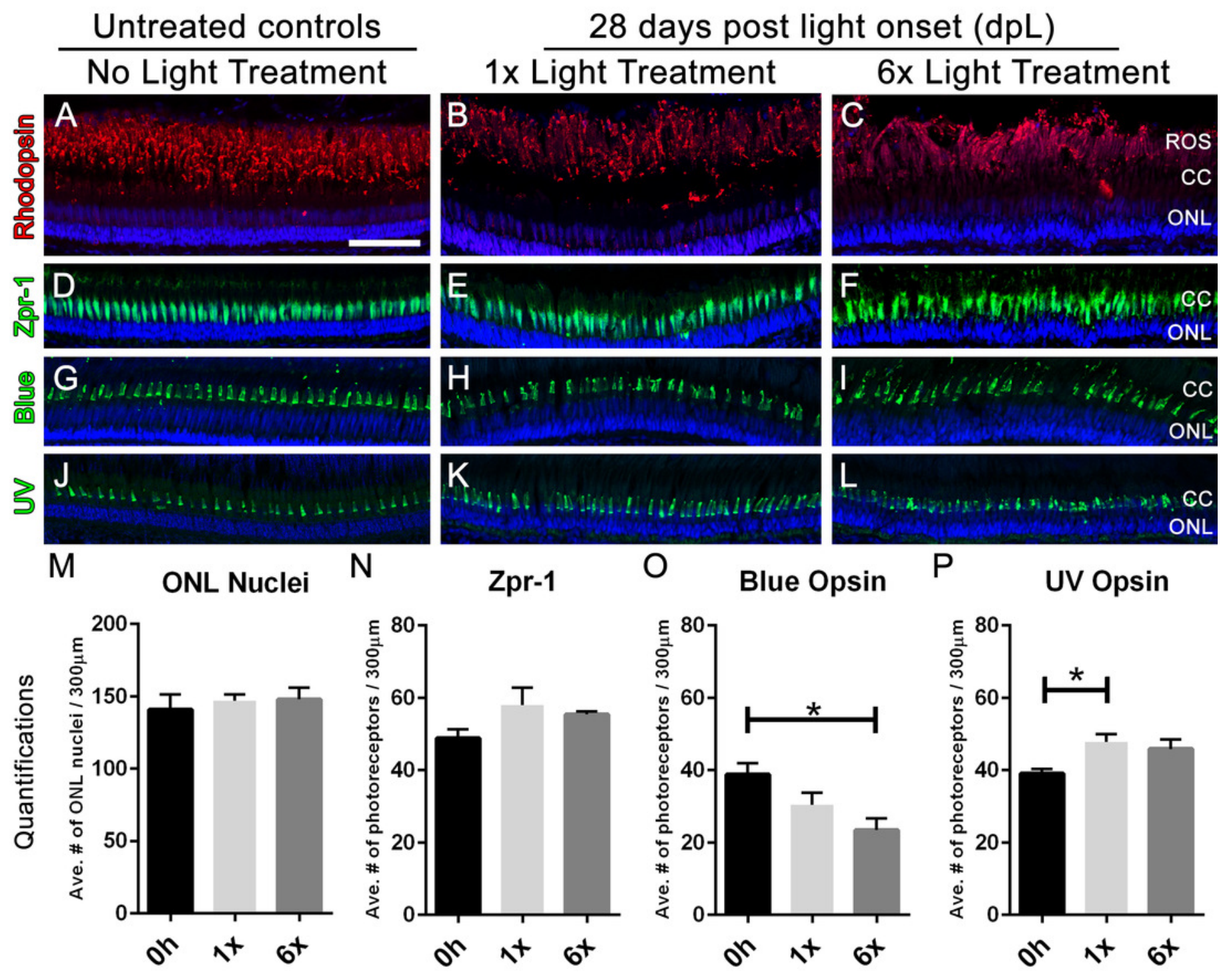




\section{Table 1 (on next page)}

Primer sequences used for RT-qPCR

All primers were designed to span introns using the NCBI/Primer-BLAST tool with a target product size of 115-150 base pairs. 


\begin{tabular}{|c|c|c|}
\hline $\begin{array}{l}\text { Target } \\
\text { Gene }\end{array}$ & Forward Primer Sequence (5'-3') & Reverse Primer Sequence (5'-3') \\
\hline cdk1 & 5'- GGT CTG GCC AGA TGT TGA GTC TC -3' & 5'- TCG TGC CGA AAT CCT CTT AGG G -3' \\
\hline$c$-jun & 5'- AGA GAC GCA GAG CGC ATG A -3' & 5'- CGC GTC CCT GTT TTA CTC CTA -3' \\
\hline pax6a & 5'- TCG AGG TTC CCT TGT TGG AC -3' & 5'- GAC GCA CGG TTC CAA GTT TC -3' \\
\hline pax6b & 5'- CCA CAC CGT ACG GGA TTC AA -3' & 5'- TCC CAG CGT CCC TCT TAT CT -3' \\
\hline pcna & 5'- TAC TCA GTG TCT GCT GTG GTT TCC -3' & 5'- CAT TTA ATA AGT GCG CCC GC -3' \\
\hline shha & 5'- AGT CTT ACC TTT CGC ATC CCC -3' & 5'- GAT GTC CTT GCC GTC TCC TC -3' \\
\hline six3b & 5'- GAT AGC AGC GCA AAC ACG AC -3' & 5'- CGC GAA ATT GGG CAG GAA AA -3' \\
\hline tgif1 & 5'- CCC ATC TAT CCA CAC TAC AGG TTT -3' & 5'- CTG AGC ATT TCG CCA CCC TT -3' \\
\hline gfap & 5'- GCA GAC AGG TGG ATG GAC TCA -3' & 5'- GGC CAA GTT GTC TCT CTC GAT C -3' \\
\hline vim & 5'- TAA GCC TGC GAG AGT CCA TGA -3' & 5'- TCG TTT TGG GTG GAC TCG TT -3' \\
\hline glulB & 5'- GCC CGC TTC CTC CTA CAC A -3' & 5'- СTC CTC AAT ATG CTT CAA ACC TCC -3' \\
\hline kcnj10a & 5'- CCT GTT TTC GGC CAC CTT TG -3' & 5'- CGG CGT ATG GTT GGA TGG AG -3' \\
\hline rlbpa & 5'- CTG CGT GCC TAC TGT GTA ATC C -3' & 5'- GCT CGG TGT GTT TGA TTC CAG -3' \\
\hline rlbpB & 5'- TGA GAC GGA TGA GAA GCG AA -3' & 5'- ACC TCA CAA GCA CGC CAT C -3' \\
\hline stat3 & 5' - GAG GAG GCG TTT GGC AAA -3' & 5'- TGT GTC AGG GAA CTC AGT GTC TG -3' \\
\hline
\end{tabular}

2 Table 1. Primer sequences used for RT-qPCR. All primers were designed to span introns using

3 the NCBI/Primer-BLAST tool with a target product size of 115-150 base pairs. 
